\section{P-360 IS OCCUPATIONAL EXPOSURE TO POLYCYCLIC AROMATIC HYDROCARBONS ASSOCIATED WITH PROSTATE CANCER RISK? RESULTS FROM PROTEUS, MONTREAL, CANADA}

${ }^{1}$ Christine Barul, Marie-Elise Parent. ${ }^{1}$ Centre Armand-Frappier Santé Biotechnologie, Institut national de la recherche scientifique, Canada

\subsection{6/OEM-2021-EPI.287}

Introduction Polycyclic aromatic hydrocarbons (PAHs) have been shown to have endocrine-disrupting effects. Exposure could be carcinogenic to the prostate, a hormone-dependent organ.

Objective To examine the association between lifetime occupational exposure to PAHs and prostate cancer risk.

Methods Face-to-face interviews elicited detailed work histories for 1,937 histologically confirmed prostate cancer cases (524 aggressive) and 1,994 controls from Montreal, Canada. Industrial hygienists applied the hybrid expert assessment approach to assign concentration, frequency and certainty of exposure to benzo(a)pyrene, PAHs from different sources (wood, coal, petroleum, other sources) and PAHs from any source. Odds ratios (ORs) adjusted for age, ancestry, education, lifestyle and occupational factors, and their 95\% confidence intervals (CI), were estimated using unconditional logistic regression.

Results After restriction to probable and definite exposure, and application of a 5-year lag, no clear association was observed between exposure to PAHs and overall prostate cancer risk, although a weak positive dose-response pattern emerged for total duration of exposure to PAHs from wood $(\mathrm{OR}=1.06$; 95\%CI 0.95 to 1.18 , per 5 -year increment). Men in the upper tertile of cumulative exposure to these PAHs showed an elevated OR of 1.54 (95\%CI 0.60 to 3.92), predominantly reflecting the non-aggressive form of the tumour $(\mathrm{OR}=1.74 ; \mathrm{OR}=0.67$ to 4.56$)$. Nevertheless, exposure to PAHs from wood occurring at least 10 years before the index date was associated with some elevated risks of high-grade tumours $(\mathrm{OR}=1.54,95 \% \mathrm{CI}$ 0.70-3.39). Similar results were found in sensitivity analyses, including those restricted to controls recently screened for prostate cancer or in those prioritizing the Gleason score ascertained from prostatectomy over that from biopsy.

Conclusion Only a handful of small studies have used detailed exposure assessment protocols to study the role of PAHs in prostate cancer risk. Our findings provide some limited evidence that exposure to PAHs from wood increases the risk of prostate cancer.

\section{P-361 OCCUPATIONAL EXPOSURE TO ENGINE EXHAUST AND PROSTATE CANCER RISK}

${ }^{1}$ Christine Barul, Marie-Elise Parent. ${ }^{1}$ Centre Armand-Frappier Santé Biotechnologie, Institut national de la recherche scientifique, Canada

\subsection{6/OEM-2021-EPI.288}

Introduction Some engine exhaust components are carcinogenic or have hormone-disrupting properties.

Objective To investigate the association between lifetime occupational exposure to various engine exhausts (EE) and prostate cancer risk.

Methods In the context of a case-control study conducted in Montreal, Canada, 1,924 histologically-confirmed prostate cancer cases (436 aggressive) and 1,989 population controls were recruited. Complete occupational history, socio-demographic and lifestyle factors were collected during in-person interviews. Industrial hygienists conducted semi-quantitative evaluations of intensity, frequency and reliability of exposure to leaded and unleaded gasoline EE, any diesel EE, heavy diesel EE, light diesel EE, jet fuel EE, and propane $\mathrm{EE}$ in each job held $\geq 2$ years. Odds ratios (ORs) adjusted for age, ancestry and education, and 95\% confidence intervals (CI), were estimated with unconditional logistic regression, modelling the association between each EE and prostate cancer risk.

Results Ever exposure to leaded gasoline EE was associated with a slight increase in risk of overall prostate cancer $(\mathrm{OR}=1.13,95 \% \mathrm{CI} 0.98$ to 1.31$)$, after restricting to probable and definite exposures, and applying a 5-year lag. Although no formal statistical heterogeneity in risks appeared, the association was slightly stronger for non-aggressive cancers than aggressive ones. No dose-response relationships emerged for total duration or cumulative exposure. Men who had ever been exposed to jet fuel EE showed a decreased odds of the tumor $(\mathrm{OR}=0.3495 \% \mathrm{CI} 0.19$ to 0.61$)$. No association was found with exposure to any other EE. Mutual adjustments for other EE and sensitivity analyses restricting controls to men recently screened for prostate cancer yielded results consistent with the main findings.

Conclusion We found limited evidence for a deleterious role of occupational exposure to leaded gasoline $\mathrm{EE}$ in the development of prostate cancer. This is the first study to examine the distinct role of leaded and unleaded gasoline $\mathrm{EE}$ in the etiology of this cancer.

\section{P-362 GENDER DIFFERENCES IN SKIN RELATED QUALITY OF LIFE}

${ }^{1}$ Amira Omrane, Asma Kheder, Harrathi Chayma, Maher Maoua, Lamia Bouzgarrou, Taoufik Khalfallah, Najib Mrizak, Mohamed Akrout, Adnene Hanchi, Hichem Belhadj Ali. ${ }^{7}$ Faculty of Medicine of Monastir, Tunisia

\subsection{6/OEM-2021-EPI.289}

Background Quality of life (QL) is an important component of individuals' general well-being, particularly in active adults. However, factors influencing skin related QL have not been fully examined. Furthermore, the role of gender differences in relation to QL in occuptaional dermatitis (OD) has also not been examined in detail. This study aimed to assess determinants of skin-related QL of healthcare workers and examine the role of gender differences.

Methods A cross-sectional and exhaustive study was conducted among healthcare workers of four public hospitals in the central region of Tunisia. All cases of dermatitis recognized as occupational disease were included. Skin-related QoL was assessed using the validated Tunisian version of the 'Dermatology Life Quality Index' (DLQI).

Results A total of 37 cases of OD were collected with an annual incidence of 4.2 cases per 10,000 workers. The population was predominantly female (73\%) and mean aged 44.7 \pm 9.4 years. Nurses were the most represented occupational category (38\%). Allergic contact dermatitis was the most frequent diagnosis (96\%). The median score of DLQI was 5. Twelve patients $(32 \%)$ had a DLQI score $>10$, meaning a significant impairment in skin related QoL. Among them, 11 $(92 \%)$ were female $(p=0.11)$. Multivariate analysis showed an association between the impairment of skin-related QoL and female gender $(\mathrm{p}=0.04 ; \mathrm{OR}=19.384)$, exposure to 